\title{
Cesium desorption intensity at the river-sea interface
}

\author{
A. Delaval ${ }^{1}$, C. DufFA $^{1}$, O. RADAKOVITCH ${ }^{1 *}$
}

${ }^{1}$ IRSN, PSE/ENV, 13115 Cadarache, France (adrien.delaval@irsn.fr; celine.duffa@irsn.fr; olivier.radakovitch@irsn.fr)

In case of nuclear accident, a significant source of radiocesium could be transported to the ocean by river particles contaminated from land deposited fallout, or from releases within the catchments. As an alkalkine earth metal, Cs is weakly bound to particles and can be released from solid to dissolved phase during the transition from freshwater to seawater. Many studies and experimental designs have been conducted on particle samples from different areas of the world in order to characterize the range and timescale of this desorption process. They have shown a large variation on the proportion released as well as on the kinetic rates observed, but there is still no consensus on the intensity of this process. A review of world litterature was performed in order to obtain a better estimation of released cesium at the fresh-seawater interface and to characterize the parameters governing the desorption (32 papers). Desorption starts at a low salinity (3-4) and reach a threshold around 15 , but a wide range of desorbed fraction (0-86\%) was observed, in relation with the experimental design. For particles containing radiocesium due to an environmental contamination, the range was 0 to $64 \%$ with a peak at $3 \%$. In contrast, desorption of particles contaminated in laboratory varied between 6 and $86 \%$ with a multimodal distribution. This discrepancy can be associated to an "aging" effect in the environment as well as to the nature of the particles, but it must be also influenced by the solution chemistry, adsorbed activity and contact time with the spike for the laboratory studies. Finally, two first-order kinetics are observed, the first one with a half-life reaction rate of one hour. 
This abstract is too long to be accepted for publication.

Please revise it so that it fits into the column on one page. 